\title{
Modelling of Heated Composite Floor Slabs with Reference to
}

\author{
the Cardington Experiments
}

February 15, 2002

\begin{abstract}
This paper describes a method of modelling composite floor slabs in fire conditions using a stressresultant approach. The FEAST suite, which consists of two main computer programs is described. The first, SRAS, is designed to model the behaviour of arbitrary orthotropic plate sections at elevated temperatures. The second program, FEAI, interfaces with the finite element package ABAQUS, allowing realistic models of the behaviour of whole structures in fire conditions to be obtained. The paper describes how SRAS was used to analyse the floor slab of the Cardington fire tests and results showing the behaviour of the slab under a variety of loading conditions are presented. The suitability of FEAI as a key component in the analysis of redundant structures under fire conditions is briefly demonstrated.
\end{abstract}

\section{Introduction}

A primary concern of fire safety engineering is to prevent the catastrophic collapse of structures due to fires. The purpose is to ensure that the occupants of a building can escape safely from a fire, without being trapped by the building collapsing. Fire-fighters also need to be protected from such a possibility. 
The research described in this paper was initiated by the widespread recognition that the traditional methods of design for ensuring the fire safety of composite steel framed structures oversimplify the problem and produce expensive, highly conservative, designs. The traditional approach centred on testing individual structural elements in isolation and then applying the results from these tests to entire structures. This approach had the severe drawback of applying the results from determinate, or nearly determinate, structures in test furnaces to highly redundant real life buildings. Recently there has been a move towards the much more realistic approach of trying to predict the behaviour of whole structures in fire conditions. Such an approach allows the high degree of redundancy of most structures to be considered in fire safety design.

To allow the behaviour of an entire structure in fire conditions to be studied, a series of tests were performed on a full scale, eight storey, steel-framed building at Cardington, Bedfordshire in $1995 .^{10}$ These experiments produced a huge amount of experimental data that can be used to develop a detailed understanding of the physical phenomena which dominate structural behaviour under fire loading. The purpose of this paper is to present a numerical modelling technique which is helpful in gaining some of this understanding.

The most demanding aspect of modelling composite steel-framed structures is the modelling of the reinforced concrete floor slabs. Numerical modelling of reinforced concrete is challenging because the stress-strain relationship is highly non-linear and is different in tension and compression. The behaviour is particularly complex when trying to model heated concrete slabs with a high degree of edge restraint. This paper describes an approach used for modelling the concrete floor slabs in the Cardington tests and the results obtained for the strength and behaviour of the slabs. The modelling was performed using the FEAST (Finite Element Analysis of Shells at High Temperatures) suite of programs. FEAST was developed specifically for the analysis of complex orthotropic plates such as the Cardington floor slab. It was also designed to interact smoothly with the commercial finite element package, ABAQUS.

The first section of the paper provides a general description of the approaches available for modelling plate structures at high temperatures and a description of the software developed during the course 
of this research to model such structures. The second section contains a detailed description of how FEAST was used to model the Cardington slab. A description of the assumptions made during the modelling is given. In the final section the results obtained regarding the behaviour of the Cardington slab are presented. These results provide valuable information about the behaviour of the slab in isolation. This information will be used in subsequent papers when a description of the behaviour of the whole Cardington structure during the fire tests will be given. To demonstrate the validity of the software developed during this research a comparison of numerical results with experimental data from the first Cardington fire test is also presented.

\section{Modelling of Plates}

\subsection{Depth Integration Approach to Modelling Plates}

The usual approach to modelling materially non-linear plate structures using finite element techniques is to use what can be described as the depth integration technique. This method calculates the stresses through the depth of an element by consideration of the geometry of the element, its strain state and its material properties. The accuracy of a calculation using this technique can be increased by increasing the number of points through the depth of the plate at which the stresses are calculated. The integration of the stresses to obtain forces is normally done using Simpson's rule. ${ }^{8}$

After a number of attempts to model the Cardington slab using the depth integration approach with the concrete model built in to ABAQUS, it was found that numerical problems were too severe for results to be obtained. Although it was a little difficult to determine the exact source of the numerical problems, it was clear that the plasticity algorithm was failing to converge. Others who have attempted this approach to modelling complex plates, particularly at elevated temperatures, have had similar difficulties. This has usually meant that results have either only been available for a limited range of plate behaviour; or that the models used have been very simplistic in terms of material behaviour; ${ }^{1,3}$

or some other factor, such as geometric non-linearity, ${ }^{3,14,15}$ has not been included in the analysis. 


\subsection{Stress-Resultant Approach to Modelling Plates}

An alternative method of plate modelling is to use a stress-resultant approach. This essentially involves the combination of the material behaviour and geometry of a plate into one set of equations. The forces and moments per unit width of plate are calculated based on the strain, curvature and temperature of the plate reference surface. Temperature gradients can also be taken account of. The drawback of this method is that it does not allow stresses to be output from the analysis; only stress-resultants. This means that it is not easy to determine the variation of stresses through the depth of the plate. Despite this restriction, the method is very powerful in that it allows the analyst to define a plate with arbitrary behaviour without the need to resort to complex multi-axial material models with their associated numerical convergence problems.

For a linear-elastic unheated orthotropic plate the relationship between stress-resultants and generalised strains can be represented by:

$$
\left[\begin{array}{c}
P_{11} \\
P_{22} \\
P_{12} \\
\hline M_{11} \\
M_{22} \\
M_{12}
\end{array}\right]=\left[\begin{array}{lll|lll}
K_{11} & K_{12} & K_{13} & K_{14} & K_{15} & K_{16} \\
K_{21} & K_{22} & K_{23} & K_{24} & K_{25} & K_{26} \\
K_{31} & K_{32} & K_{33} & K_{34} & K_{35} & K_{36} \\
\hline K_{41} & K_{42} & K_{43} & K_{44} & K_{45} & K_{46} \\
K_{51} & K_{52} & K_{53} & K_{54} & K_{55} & K_{56} \\
K_{61} & K_{62} & K_{63} & K_{64} & K_{65} & K_{66}
\end{array}\right]\left[\begin{array}{c}
\epsilon_{11} \\
\epsilon_{22} \\
\epsilon_{12} \\
\hline \phi_{11} \\
\phi_{22} \\
\phi_{12}
\end{array}\right]
$$

The vector on the left of Eqn. 1 is a set of stress resultants for the plate representing two direct membrane forces, an in-plane shear force, two bending moments and a twisting moment. The vector on the right contains the corresponding generalised strains. The matrix contains a set of stiffness terms.

The degree to which the matrix is populated increases as more complex plates are modelled. For the simplest cases it is sufficient to populate only the leading diagonal of the matrix, and possibly some off diagonal terms to allow for Poisson effects. However, for most cases, it is necessary to at least partially populate the off-diagonal quadrants of the matrix to introduce coupling between membrane 
and bending actions and to allow formulations for more complex plates to be produced. If this coupling is introduced, it is necessary to select a plate reference surface at which a pure membrane force is deemed to act and from which all quantities can be measured. Typically this might coincide with the top or bottom surface of the plate, or pass through the centroid of the cross section. Eqn. 1 can be further generalised by the addition of two transverse (vertical) shear terms.

For a plate with non-linear material behaviour, the terms in the stiffness matrix vary with the strain state. In such cases Eqn. 1 is modified into the incremental form:

$$
\left[\begin{array}{c}
\delta P_{11} \\
\delta P_{22} \\
\delta P_{12} \\
\hline \delta M_{11} \\
\delta M_{22} \\
\delta M_{12}
\end{array}\right]=\left[\begin{array}{lll|lll}
K_{11} & K_{12} & K_{13} & K_{14} & K_{15} & K_{16} \\
K_{21} & K_{22} & K_{23} & K_{24} & K_{25} & K_{26} \\
K_{31} & K_{32} & K_{33} & K_{34} & K_{35} & K_{36} \\
\hline K_{41} & K_{42} & K_{43} & K_{44} & K_{45} & K_{46} \\
K_{51} & K_{52} & K_{53} & K_{54} & K_{55} & K_{56} \\
K_{61} & K_{62} & K_{63} & K_{64} & K_{65} & K_{66}
\end{array}\right]\left[\begin{array}{c}
\delta \epsilon_{11} \\
\delta \epsilon_{22} \\
\delta \epsilon_{12} \\
\hline \delta \phi_{11} \\
\delta \phi_{22} \\
\delta \phi_{12}
\end{array}\right]
$$

The stiffness matrix now contains instantaneous tangent values of stiffness, and both vectors represent small changes from the existing state. It is possible to generalise this equation further by taking into account the differences between total, mechanical and thermal strains. Typically in numerical work the total strains and the temperature state in plates are known. As it is the mechanical strains that produce stresses, and hence forces and moments, it is these that must be used in Eqn. 2. This gives 
rise to the following equation:

$$
\left[\begin{array}{c}
\delta P_{11} \\
\delta P_{22} \\
\delta P_{12} \\
\delta M_{11} \\
\delta M_{22} \\
\delta M_{12}
\end{array}\right]=\left[\begin{array}{lll|lll}
K_{11} & K_{12} & K_{13} & K_{14} & K_{15} & K_{16} \\
K_{21} & K_{22} & K_{23} & K_{24} & K_{25} & K_{26} \\
K_{31} & K_{32} & K_{33} & K_{34} & K_{35} & K_{36} \\
\hline K_{41} & K_{42} & K_{43} & K_{44} & K_{45} & K_{46} \\
K_{51} & K_{52} & K_{53} & K_{54} & K_{55} & K_{56} \\
K_{61} & K_{62} & K_{63} & K_{64} & K_{65} & K_{66}
\end{array}\right]\left[\begin{array}{c}
\delta \epsilon_{\mathrm{t} 11}-\delta \epsilon_{\mathrm{T} 11} \\
\delta \epsilon_{\mathrm{t} 22}-\delta \epsilon_{\mathrm{T} 22} \\
\delta \epsilon_{\mathrm{t} 12}-\delta \epsilon_{\mathrm{T} 12} \\
\hline \delta \phi_{\mathrm{t} 11}-\delta \phi_{\mathrm{T} 11} \\
\delta \phi_{\mathrm{t} 22}-\delta \phi_{\mathrm{T} 22} \\
\delta \phi_{\mathrm{t} 12}-\delta \phi_{\mathrm{T} 12}
\end{array}\right]
$$

where: $\delta \epsilon_{\mathrm{t} i j}=$ an increment of total strain

$$
\begin{gathered}
\delta \phi_{\mathrm{t} i j}=\text { an increment of total curvature } \\
\delta \epsilon_{\mathrm{T} i j}=\text { an increment of thermal strain } \\
\delta \phi_{\mathrm{T} i j}=\text { an increment of thermal curvature }
\end{gathered}
$$

The values of thermal strains and curvatures in Eqn. 3 can easily be determined provided that the coefficient of thermal expansion for the plate is known. Thermal strain is given by:

$$
\alpha_{(T)}=\frac{\epsilon_{t h(T)}}{\Delta T}
$$

where: $\epsilon_{t h(T)}=$ the strain resulting from a change in temperature

$$
\begin{aligned}
\Delta T & =\text { change in temperature } \\
\alpha_{(T)} & =\text { coefficient of thermal expansion at temperature } \mathrm{T} \\
T & =\text { temperature }
\end{aligned}
$$

If it is assumed that the temperature gradient through the slab is linear then the thermal curvatures 
can be calculated from:

$$
\phi=\alpha G
$$

where: $\mathrm{G}=$ temperature gradient through slab

Eqn. 3 can be used incrementally during a finite element analysis to provide descriptions of the behaviour of arbitrary plates under complex loading conditions.

\subsection{The FEAST Suite of Programs}

The FEAST suite was developed to provide a set of programs that enabled the modelling of arbitrary plates at high temperatures using the stress-resultant approach to be undertaken. It consists of two main modules:

- SRAS - Stress-Resultant Analysis of Shells. This program analyses user defined plates over an arbitrary range of stress-strain-temperature states.

- FEAI - Finite Element Analysis Interface. This program allows stress-resultant based calculations for user-defined plates to be undertaken with ABAQUS.

Although the FEAST suite was developed mainly for modelling the Cardington slab, care was taken to ensure that the programs could be easily adapted to model any plate. No limitations were placed on the number of materials that could be included in a plate section or on the geometry of the section. All input parameters were defined externally in separate files so that changing between plate sections was straightforward. This generality ensures that FEAST can be used in other problems where complex plates needed to be modelled. To be consistent with this approach FEAST is described in this section in general terms and then its specific application to the Cardington slab is described in the following section. 


\subsection{SRAS}

SRAS was written to determine the behaviour of plates over a wide range of strain-curvature-temperature states and to provide output that could easily be manipulated for graphical representation. To calculate a plate's behaviour SRAS reads an input file that divides the plate's cross section into a number of layers as shown in Fig. 3. Each layer is defined by its area, the distance of its centroid from the plate reference surface and its material. Stress-strain-temperature data-files are read for each material in the cross-section. The input file contains user-specified information on the range of strains, curvatures and temperatures over which the stress-resultants are to be calculated and also the intervals between these values.

Based on the assumption that initially plane sections remain plane after bending the strain in a given layer is calculated according the equation:

$$
\epsilon_{l}=\epsilon_{r}+z_{l} \phi
$$

where: $\epsilon_{l}=$ average strain in the layer

$$
\begin{aligned}
& \epsilon_{r}=\text { reference surface strain } \\
& z_{l}=\text { distance of the centre of a layer from the reference surface } \\
& \phi=\text { curvature of the reference surface }
\end{aligned}
$$

The temperature in each layer depends on the reference surface temperature and the thermal gradient through the plate. SRAS allows for non-linear gradients but requires that they can be approximated by a polynomial that relates the distance from the reference surface ( $x$-variable) to temperature ( $y$-value). The form of the temperature profile in a plate may change with increasing reference surface temperature. This is allowed for by permitting the user to define a number of polynomial relationships that correspond to the temperature profiles at various reference surface temperatures, linear interpolation is used between these profiles. The layers of the plate may be divided into groups with different sets of polynomial temperature relationships. This capability is useful when dealing 
with plates of varying thickness as shown in Fig. 3.

Once the strain and temperature in each layer have been established the stress can be determined from the stress-strain-temperature relationship of the layer's material. Given the stress in a layer it is possible to establish the force by multiplying the stress by the layer's area. The force in each direction in a plate section is the integral of the stresses over the area of the section:

$$
F=\int_{A} \sigma d A
$$

which in the SRAS layered approach reduces to:

$$
F=\sum_{i=1}^{n} A_{i} \sigma_{i}
$$

where: $F=$ the force acting on the cross section

$$
\begin{aligned}
& A=\text { the area of the cross section } \\
& A_{i}=\text { the area of layer } \mathrm{i} . \\
& n=\text { the number of layers }
\end{aligned}
$$

Similarly the moment about the reference surface is calculated from the expression:

$$
M_{r}=\int_{A} \sigma z d A
$$

which in the SRAS layered approach reduces to:

$$
M_{r}=\sum_{i}^{n} F_{i} z_{i}
$$

where: $M_{r}=$ moment about the reference surface

$$
F_{i}=\text { force in layer } i
$$

The moments and forces produced by this procedure are converted into stress-resultants by dividing 
by the width of plate section that was used in the analysis.

A flow diagram describing the calculation sequence in SRAS is shown in Fig. 1 and the details of how the stress in each layer is calculated are shown in Fig. 2.

SRAS can be made to output data in a number of forms. Most simply it can produce tabulated data for plotting the force-strain and moment-curvature behaviour of plates but it can also produce output that can be plotted as force-moment interaction diagrams for plates.

\subsection{FEAI}

FEAI takes advantage of the fact that ABAQUS can be programmed to model plates using a stressresultant approach with the addition of a user- defined subroutine. At the start of each load increment during an analysis, ABAQUS calls FEAI and passes to it information about the current state of the plate section. This information includes the current temperature of the plate reference surface, $\mathrm{T}$; a vector, $\mathbf{N}_{\mathbf{0}}$, containing section stress-resultants; a vector, $\mathbf{E}$, containing section total strains and a vector, $\Delta \mathbf{E}$, containing section total strain increments for the current iteration of the load increment. ABAQUS also allows the user to pass information from the problem's ABAQUS input file directly to FEAI. In FEAI this information includes the thermal expansion coefficient of the plate, the final thermal gradient through the plate and its Poisson ratio. FEAI must perform two functions with this data: it must update the stress-resultants to their values at the end of the load increment and it must provide a section stiffness matrix, $\partial \mathbf{N}_{\mathbf{0}} / \partial \mathbf{E}$. The process can be represented algebraically as:

$$
\mathbf{N}_{\mathbf{u}}=\mathbf{N}_{\mathbf{0}}+\frac{\partial \mathbf{N}_{\mathbf{0}}}{\partial \mathbf{E}}\left(\Delta \mathbf{E}-\Delta \mathbf{E}_{\mathbf{T}}\right)
$$

where: $\mathbf{N}_{\mathbf{u}}=$ the updated stress-resultant vector.

$\mathbf{E}_{\mathbf{T}}=$ a vector containing section thermal strain and curvature increments

The quantity $\partial \mathbf{N}_{\mathbf{0}} / \partial \mathbf{E}$ represents the matrix of tangent stiffness terms in Eqn. 3. To calculate these stiffnesses it is necessary to take the vector $\mathbf{N}_{\mathbf{0}}$ and then calculate two other vectors $\mathbf{N}_{\delta \epsilon}$ and $\mathbf{N}_{\delta \phi}$. 
These two vectors represent the stress resultants in the slab after infinitesimal increases in the strain, $\delta \epsilon$, and curvature, $\delta \phi$, respectively. The vectors $\mathbf{N}_{\delta \epsilon}$ and $\mathbf{N}_{\delta \phi}$ are computed in a very similar manner to the way in which stress-resultants are calculated in SRAS. The only variation is that in FEAI the strains and curvatures are based on values obtained from the ABAQUS analysis rather than being specified by the user. By taking components from all three stress-resultant vectors it is possible to determine the terms of $\partial \mathbf{N}_{\mathbf{0}} / \partial \mathbf{E}$. The terms in the one direction of the plate are shown below and terms in the perpendicular direction can be obtained by altering the subscripts. The stress-resultants in perpendicular directions in the plate are coupled by making $K_{12}=\nu K_{11}$ etc.

$$
\begin{array}{ll}
K_{11}=\frac{\partial N_{0_{1}}}{\partial \epsilon_{1}}=\frac{N_{\delta \epsilon_{1}}-N_{0_{1}}}{\delta \epsilon}, & K_{14}=\frac{\partial N_{0_{1}}}{\partial \phi_{1}}=\frac{N_{\delta \phi_{1}}-N_{0_{1}}}{\delta \phi} \\
K_{44}=\frac{\partial N_{0_{4}}}{\partial \epsilon_{4}}=\frac{N_{\delta \epsilon_{4}}-N_{0_{4}}}{\delta \phi}, & K_{41}=\frac{\partial N_{0_{4}}}{\partial \phi_{1}}=\frac{N_{\delta \phi_{4}}-N_{0_{4}}}{\delta \epsilon}
\end{array}
$$

where: $N_{0_{i}}=$ is the $i$ th term in the vector $\mathbf{N}_{\mathbf{0}}$

$$
\begin{aligned}
& N_{\delta \epsilon_{i}}=\text { is the } i \text { th term in the vector } \mathbf{N}_{\delta \epsilon} \\
& N_{\delta \phi_{i}}=\text { is the } i \text { th term in the vector } \mathbf{N}_{\delta \phi}
\end{aligned}
$$

FEAI uses $1 \%$ of the total strain or curvature for values of $\delta \epsilon$ and $\delta \phi$. In this formulation the values of in-plane shear stiffness and the twisting stiffness are assumed to be the lesser of the membrane stiffnesses and bending stiffnesses respectively.

The values of the terms in the vector $\Delta \mathbf{E}_{\mathbf{T}}$ are obtained by subtracting the mechanical component from the total values using Eqns. 4 and 5. For the purposes of calculating thermal curvatures, the thermal gradients are assumed to be linear through the section and to increase linearly during the analysis from zero at the start of the heating to the value specified in the ABAQUS input file at the end. This is in contrast to the polynomial temperature profiles used for determining the material properties and hence the stiffness of the section. 


\section{Modelling the Cardington Slab}

\subsection{The Cardington Slab}

The concrete floor slab used in the Cardington frame was typical of floor slabs used in composite construction. The slab was orthotropic due to ribs running in one direction along its bottom surface. Above the ribs a layer of anti-cracking reinforcement mesh was included. The bottom surface was covered in a layer of steel decking that was bonded to the concrete by means of small protrusions. The cross section parallel to the ribs is shown in Fig. 4. The material properties of the concrete used in the slab were tested on each of the eight floors in the Cardington frame ${ }^{10}$ and the average 28-day strength was found to be $47 \mathrm{kN} / \mathrm{mm}^{2}$.

\subsection{Assumptions made during the Modelling of the Cardington Slab}

Before a detailed description of the model used to represent the Cardington slab is given the assumptions used in it will be explained and justified. During any analysis of structures, analytical or numerical, some assumptions will be made. The aim must be to make assumptions that do not affect the outcome of analysis significantly, or at least not the parts of the analysis that are of most interest. The goal of this project is to obtain an understanding of the overall behaviour of the Cardington structure under fire conditions. The assumptions made, therefore, are aimed at achieving this goal - local details of behaviour were sometimes ignored to ensure that the overall structural trends were captured in the model. The assumptions made are listed below and then explained more fully. Some of these assumptions are implicit in the FEAST suite and some are made externally to it. It is assumed that:

- That the stress-strain relationships for the constituent materials are unique.

- That the material behaviour was uniaxial

- The Cardington slab has uniform thickness and material properties throughout the area of the building being modelled. 
- That the transverse shear stiffnesses of the slab remained constant for all strain and temperature states.

Stress-strain Relationships are Unique This assumption implies that all deformations are reversible and that during unloading materials follow the same stress-strain path as they do during loading. In the post elastic range this is manifestly untrue for all materials. It can only be justified with the argument that engineering problems are principally concerned with regimes where strains are increasing. It should be noted however that in problems where large parts of a structure start to unload any model assuming unique stress-strain relationships would be invalid. An example of such a problem is the cooling of a redundant structure after a fire. In such a case the present model of the Cardington slab would be insufficient.

This assumption also implies that creep strains are either not present or that they are accounted for in the material constitutive relationships. As will be explained, the constitutive models used are based on those in the Eurocodes and these implicitly include some allowance for creep strains. ${ }^{6,7}$ In effect then, the models used for modelling the Cardington slab assume "long-term" loading. Although fires only last a few hours at most, creep effects at high temperatures occur relatively quickly and so the assumption of "long term" loading is not unreasonable.

Material Behaviour is Uniaxial In the Cardington slab the reinforcement is a mesh consisting of bars running in perpendicular directions, stressed only along their lengths. Similarly the decking can only be considered to act in the direction parallel to the ribs. This means that all the steel components of the slab were stressed uniaxially and so a uniaxial material model for the steel was justified. By contrast the concrete in the slab was subject to a complex stress state. Biaxial stressing of concrete can lead to strength enhancements of up to $20 \%$ and typically of $10 \%$ over uniaxial peak values. Ignoring this behaviour is, in effect, using a slightly weaker concrete in the model than was used in the Cardington slab. It was felt that this small inaccuracy was justified on pragmatic grounds. Producing a stress-resultant slab model that included bi-axial material behaviour would have been impractical. 
Uniform Properties It is assumed that the geometry of the section of the Cardington slab remains constant and equal to the dimensions given in the reports of the Cardington test. ${ }^{2,5,10}$ Also any variation in the strength of the materials in different areas of the building are ignored.

When slabs are being produced it is inevitable that some "ponding " occurs, that is, deflections caused by the self-weight of the slab result in concrete flowing to the centre of the bays resulting in slightly thicker slabs in these regions. Also, the tolerance achieved when pouring concrete is quite poor; attempts to ensure that the design thickness of a slab is present over the entire floor often result in the average thickness being considerably greater. Some attempt to quantify these effects for the Cardington slab was reported by Rose et al. ${ }^{11}$ and also briefly by Huang. ${ }^{14}$ Their findings show that the Cardington slab varied in thickness between $124 \mathrm{~mm}$ and $170 \mathrm{~mm}$. It was found that the average thickness was considerably more than the design thickness of $130 \mathrm{~mm}$ but that $15 \%$ of the floor was thinner than this nominal thickness.

It would clearly be impractical to have constantly varying floor slab thickness in a stress-resultant numerical model, even if reliable data were available. Consequently it has been assumed that the dimensions of the slab are equal to those in the design drawings.

The strength of the concrete used in the floor slabs varied somewhat between floors. ${ }^{10}$ No data is available for the variation in strength between different areas of individual floors. In the absence of better information it has been assumed that the strength of the concrete is equal to $47 \mathrm{kN} / \mathrm{mm}^{2}$, the 28-day average strength. Typically, the strength of concrete rises to be about $20 \%$ more than its 28-day strength after 1 year. This increase in strength was not accounted for in the analyses and so the results are more relevant to a new structure. The strength of the steel was assumed to be equal to that quoted in the design notes.

Transverse Shear Stiffnesses Remain Constant As noted in the discussion of Eqn. 1 it is possible to add two transverse shear stiffness terms to the equation thus giving an $8 \times 8$ matrix. These two terms are not passed to FEAI by ABAQUS and so can not be varied during an analysis. ABAQUS only allows the two stiffnesses to be defined as constant values in the ABAQUS input file. 
This limitation would not have a large effect on the analysis of the Cardington slabs. It is easiest to demonstrate that this is the case by taking an analogy with a linear elastic, transversely loaded cantilever. It can be shown ${ }^{9}$ that the ratio of bending deflection to shear deflection is $1: k(h / L)^{2}$, where $k$ is a constant that for most materials is around unity, $h$ is the depth of the cantilever and $L$ its length. Clearly the shearing deflections only become important for deep cantilevers. Assuming that this argument can be extended to the Cardington slab it is clear that shear deflections are unimportant. With this in mind the transverse shear stiffnesses were given very high values in all the input files so that the predicted transverse shear deflections from finite element analyses would be effectively zero. Related to this assumption is the assumption that plane sections remain plane during bending. In reality some distortion of initially plane sections would take place as a result of transverse shear deformations. The plane section assumption was implicitly present in early work on the elastic curvature of beams by Euler and Jacob Bernoulli ${ }^{12}$ and was applied to plates by Kirchoff in 1850 and to curved plates by Love in $1888 .{ }^{4,13}$ Consequently it is often referred to as the Kirchoff-Love hypothesis. It will be assumed here on the basis that because of the high transverse shear stiffnesses the distortions of initially plane sections would be very small. If it were felt necessary to include some allowance for these distortions in the model it would be possible by altering Eqn.6. The strain due to curvature could, for example, be made to vary quadratically with distance from the reference surface rather than just linearly.

\subsection{The Model of Cardington Slab Used}

In the direction of the ribs the slab was divided into layers, as required by FEAST, in the manner shown in Fig. 4. One complete rib was taken and divided in to three vertical sections, this was necessary to allow different temperature profiles to be represented in the rib section of the slab and the trough section. The reinforcing mesh was modelled using a smeared layer approach, that is, a layer of steel of area equal to the cross sectional area of the reinforcement was added. The strain range over which SRAS calculated values was -0.01 to 0.01 at an interval of 0.005 ; the curvature range was $\left.-0.00049 \mathrm{~mm}^{(}-1\right)$ to $0.005 \mathrm{~mm}(-1)$ with an interval of $\left.0.0001 \mathrm{~mm}^{(}-1\right)$ and the temperature 
range was $0 \mathrm{C}$ to $300 \mathrm{C}$ with an interval $50 \mathrm{C}$. It was felt that these values would provide data for the overwhelming majority of the strain-curvature-temperature states that the slab would be likely to adopt. The reference surface selected for the calculations was $70 \mathrm{~mm}$ above the lower surface of the ribs, this approximately coincided with the slab geometric centroid in the direction of the ribs. In the numerical model a perfect bond was assumed between the steel decking and the concrete although in reality the steel was seen to debond from the concrete at high temperatures. This assumption can be justified by noting that the steel is heated sufficiently for it to have negligible strength towards the end of the heating.

Perpendicular to the ribs the slab was modelled in a similar way although there were some noteworthy differences. The ribs were assumed to play no part in the strength in this direction because longitudinal stresses would not be able to pass from rib to rib. This assumption means that the additional stiffness that the ribs may have provided was not included in the model. The decking was also assumed not to act because of its undulations over the ribs.

The material stress-strain curves used in SRAS were obtained from the relevant Eurocodes ${ }^{6,7}$ and are shown in figures 5,6 and 9. Steel was assumed to behave in the same manner in tension and compression while concrete was taken to have a tensile strength of one tenth of its compressive strength. In FEAI the same material properties were used for the ascending branches of the stress-strain curves but beyond this point the materials were assumed to be infinitely ductile. This was necessary because the Newton-Raphson type solver used in ABAQUS is unable to track the behaviour of structures with negative stiffnesses.

The temperature profiles through the slab were obtained from the experimental data produced by British Steel. ${ }^{2}$ For a given reference surface temperature the exact vertical distribution varied slightly between tests and between areas within the same test. For example, the areas of slab directly over beams tended to have a slightly more uniform vertical temperature distribution than those in the middle of the bays. However, these variations were small and it was felt that assuming a uniform vertical temperature distribution for a given reference surface temperature for all areas of the slab was reasonable. An area of slab near the middle of a bay in the first of the Cardington experiments 
was selected to obtain representative temperature distributions.

The temperature data available gave temperature profiles through the slab at the centre of a trough and at the centre of a rib. Temperature measurements were recorded throughout the test at two minute intervals. To obtain the polynomial curves that were required by FEAST to represent the temperature profiles in the slab, the data was examined and the profiles extracted when the sensor closest to reference surface reached temperatures equal to each of the reference surface temperatures at which profiles were required. These reference surface temperatures were 10C (ambient temperature), $50 \mathrm{C}, 100 \mathrm{C}, 150 \mathrm{C}$ and $200 \mathrm{C}$. Once this data had been selected fourth order polynomial curves were fitted to all the profiles using a least squares approach and these polynomials specified in FEAST. The curves are shown in Figs. 7 and 8.

The accuracy of temperature profiles obtained parallel to the ribs using this technique was very good because precise curves could be fitted to both the trough and rib parts of the cross-section. Perpendicular to the ribs the situation was more complex because the correct temperature profile depended on whether the section being considered contained a trough or a rib. Since the overall slab behaviour was likely to be governed by the weakest part of the cross section, the temperature profiles obtained for the trough sections of the slab were applied. These profiles were correct for the parts of the slab that were over a trough and also resulted in the hottest therefore weakest section.

\section{The Behaviour of the Cardington Slab}

\subsection{The Cardington Slab Stress-Resultant Behaviour}

The behaviour of the Cardington slab as determined by SRAS in terms of its strain-curvaturetemperature behaviour is shown in figures 10 to 12 . As might be expected given the material properties of the slab, it can be seen from Fig. 10 that there is a decrease in membrane strength and an increase in ductility as temperature increases. The large amount of ductility in the tensile region can be attributed to the contribution of the steel reinforcement and decking.

Moment-curvature behaviour is shown in Fig. 11, and again the ductility can be seen to increase 
and the strength decrease with rising temperature. It is noticeable that for positive curvatures the initial bending stiffness of the section remains roughly constant with increasing temperature but for negative curvatures it decreases. This can be explained by noting that positive bending is largely resisted by the concrete in the top of the slab and even for high reference surface temperatures this remains fairly cold and so its stiffness remains almost constant. Negative bending is resisted by the concrete in the lower portion of the slab and it is this region that becomes very hot, and so less stiff, at higher reference surface temperatures.

Moment against strain is plotted in Fig. 12 and the coupling between curvature and strain is clearly visible. For positive strains the moments arise largely from the from the tensile capacity of the steel. This is because the concrete loses almost all its strength (due to cracking) at low strains and so has very little effect on the slab behaviour. All the steel is below the reference surface and therefore gives rise to positive (sagging) moments. The form of the curve produced can be seen to resemble the steel stress-strain curve closely. Peak compressive moments are shown to increase under increasing temperature, a slightly counter-intuitive result. As with the moment-curvature behaviour, this can be explained by remembering that the bottom of the slab is heated more quickly than the top. At ambient temperatures the moment due to compressive strain results largely from the different areas of concrete providing resistance above and below the neutral surface. If the slab were to be heated uniformly then this moment would decrease since the areas would remain the same but the forces acting would reduce because of loss of material properties. Whilst this effect no doubt exists in the Cardington slab it is swamped by the temperature gradient causing the concrete in the bottom half of the slab to lose strength very much more rapidly than that in the top half, thus increasing the couple about the reference surface and hence the moment over the section. At low temperatures with low compressive strains the moment can be seen to be influenced by the steel behaviour. At ambient temperature this effect is sufficient for the hogging moments to be present until after the steel yields. The membrane forces produced by applied curvatures are shown in Fig. 13. Both positive and negative bending produce compressive forces, as would be expected given concrete's asymmetric material behaviour. The forces produced by sagging bending are slightly greater than those that result from 
hogging bending because there is a greater area of concrete above the reference surface than below it.

\subsection{Cardington Test 1}

The behaviour of the Cardington structure during the fire-tests was extremely complex and will be the subject of subsequent papers. However, to demonstrate the suitability of FEAI for such modelling, a simple comparison of the experimental deflection and the deflection predicted by ABAQUS using FEAI for the first of the Cardington tests is presented in Fig. 15. This test consisted of heating a secondary beam and the surrounding area of floor slab in an internal bay of the Cardington frame. A sketch of the area modelled is shown in Fig. 14. Because of the high degree of lateral restraint provided by the surrounding cold structure it was possible to model the test accurately by assuming a symmetry condition at mid-span of the tested beam. The boundary conditions at the other edges of the model were also assumed to be symmetrical. Non-linear geometrical effects were fully accounted for in the analysis. It is clear that not only is the final deflection predicted accurately but that the various trends and changes of rate of deflection seen in the experiment are also captured in the numerical model.

\section{Conclusions}

This investigation has demonstrated the suitability of the stress-resultant approach for analysing and modelling the behaviour of concrete floor slabs at high temperatures. The method is clearly capable of analysing complex plates and predicting their behaviour. It has been shown that modelling coupling between membrane and bending forces is important for some strain states.

It has also been shown that using stress-resultant techniques with the finite element method enables complex problems to be solved with more rigour than by using other techniques. The FEAST model that has been developed is the most sophisticated model to date that is capable of modelling the behaviour of composite structures in fire conditions. It captures many effects such as geometrical non-linearity and coupling of forces and moments that have been absent from previous models. ${ }^{1,14,15}$ 
Further work is needed to analyse the Cardington tests thoroughly using the method that has been presented.

\section{References}

[1] Sanad A.M., Rotter J.M., Usmani A.S., and O'Connor M.A. Finite element modelling of fire tests on the Cardington composite building. In Proceedings Interflam'99, volume 2, 1999.

[2] Kirby B.R. The behaviour of a multi-storey steel-framed building subjected to fire attack, experimental data. Technical report, British Steel, 1998.

[3] Bailey C.G., Burgess I.W., and Plank R.J. Computer simulation of a full-scale structural fire test. The Structural Engineer, 74(6):93-100, March 1996.

[4] Calladine C.R. Theory of Shell Structures. Cambridge University Press, 1983.

[5] Martin D.M., Kirby B.R., and O'Connor M.A. Behaviour of a multistorey, steel-framed building subjected to natural fire effects, final report. Technical report, British Steel, 1998. Confidential report.

[6] ENV. Eurocode 2, Design of Concrete Structures, 1992.

[7] ENV. Eurocode 3, Design of Composite Steel and Concrete Structures, 1994.

[8] Hibbet, Karlson and Sorenson, Providence, Rhode Island, USA. ABAQUS Users' Manual, Vols I to III, Ver 5.8, 1998.

[9] Case J., Lord Chilver, and Ross C.T.F. Strength of Materials and Structures. Edward Arnold, 3rd edition, 1992.

[10] Bravery P.N.R. Cardington large building test facility. Technical report, Building Research Establishment, 1993.

[11] Rose P.S., Burgess I.W., Plank R.J., and Bailey C.G. The influence of floor slabs on the structural behaviour of composite frames. In Lee, editor, Structures in the New Millennium, 1997. 
[12] Timoshenko S. History of Strength of Materials. Mc Graw-Hill Publishing Company, 1953.

[13] Timoshenko S. and Woinowsky-Krieger S. Theory of Plates and Shells. Mc Graw-Hill International Editions, 1959.

[14] Huang Z., Burgess I.W., and Plank R.J. Nonlinear analysis of reinforced concrete slabs subjected to fire. ACI Structural Journal, 96:127-135, Jan-Feb 1999.

[15] Huang Z., Burgess I.W., and Plank R.J. Three-dimensional modelling of two full-scale fire test on a composite building. Proceedings of the Institute of Civil Engineers, Structures and Buildings, 134(1):243-255, 1999. 


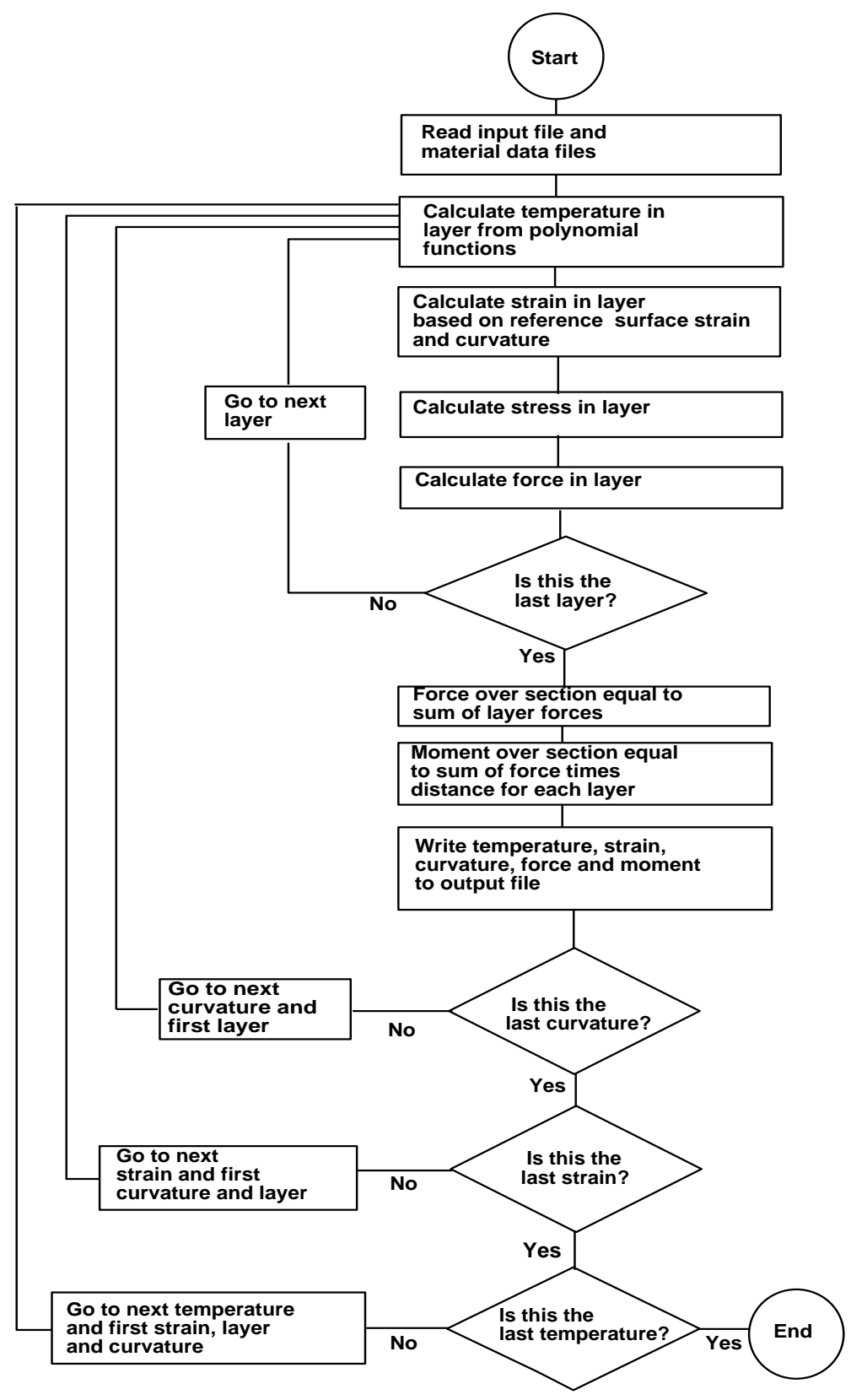

Figure 1: Flowchart describing the program SRAS 


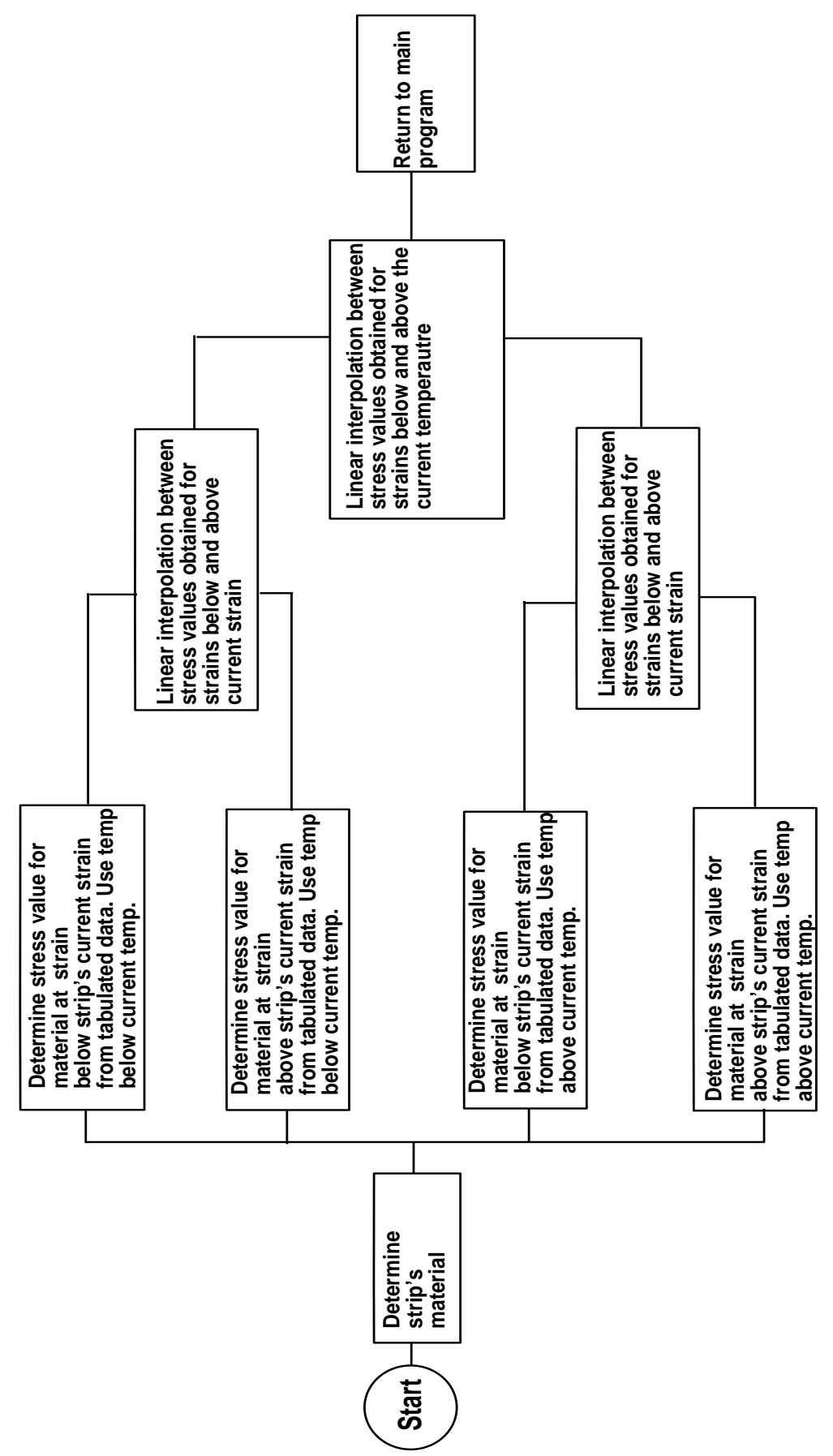

Figure 2: Flowchart describing the details of stress calculation within SRAS 
Arbitary plate section divided into a suitable number of layers
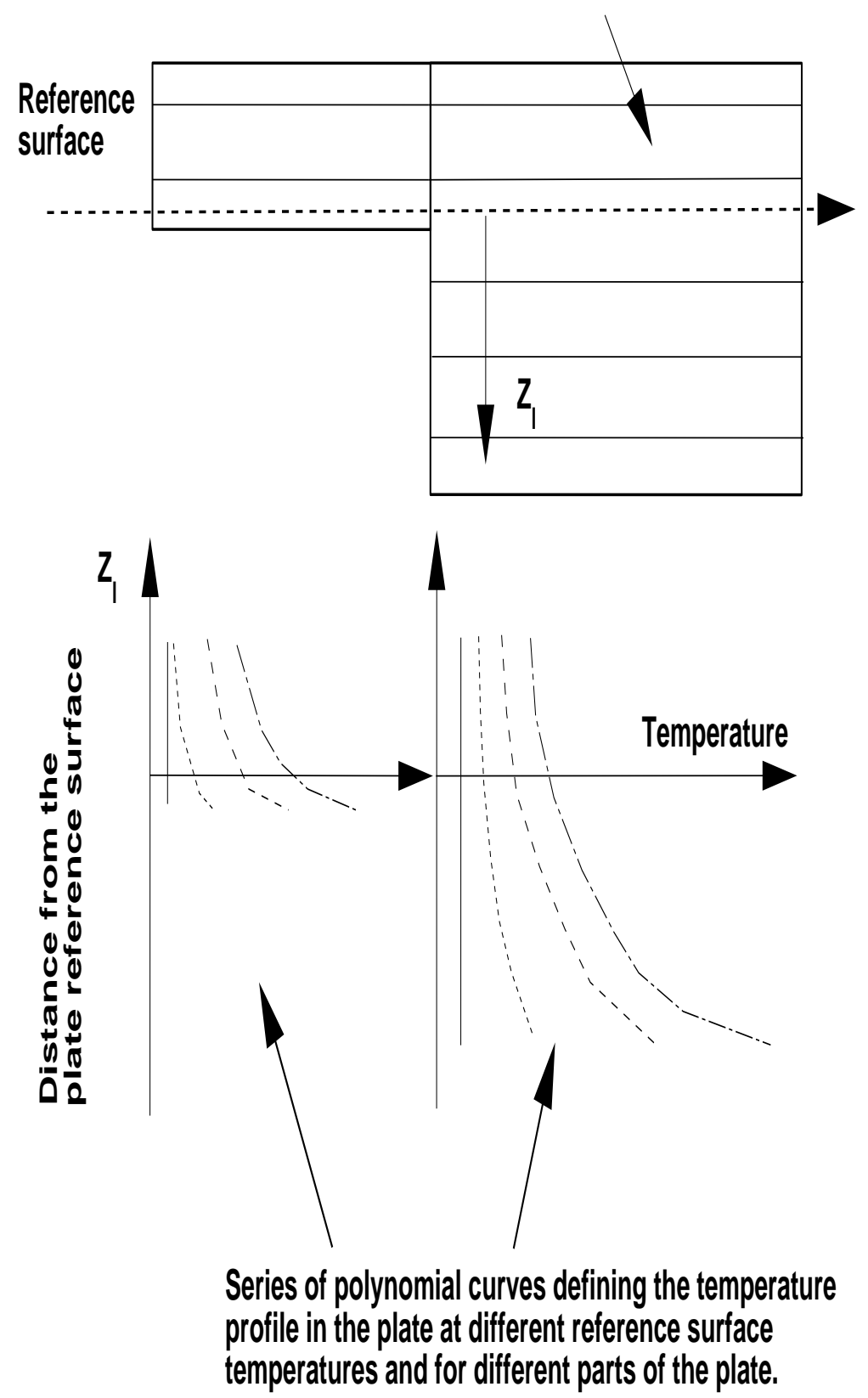

Figure 3: Diagram showing the method used by SRAS for calculating the stresses and temperatures for each layer of an arbitrary plate section 

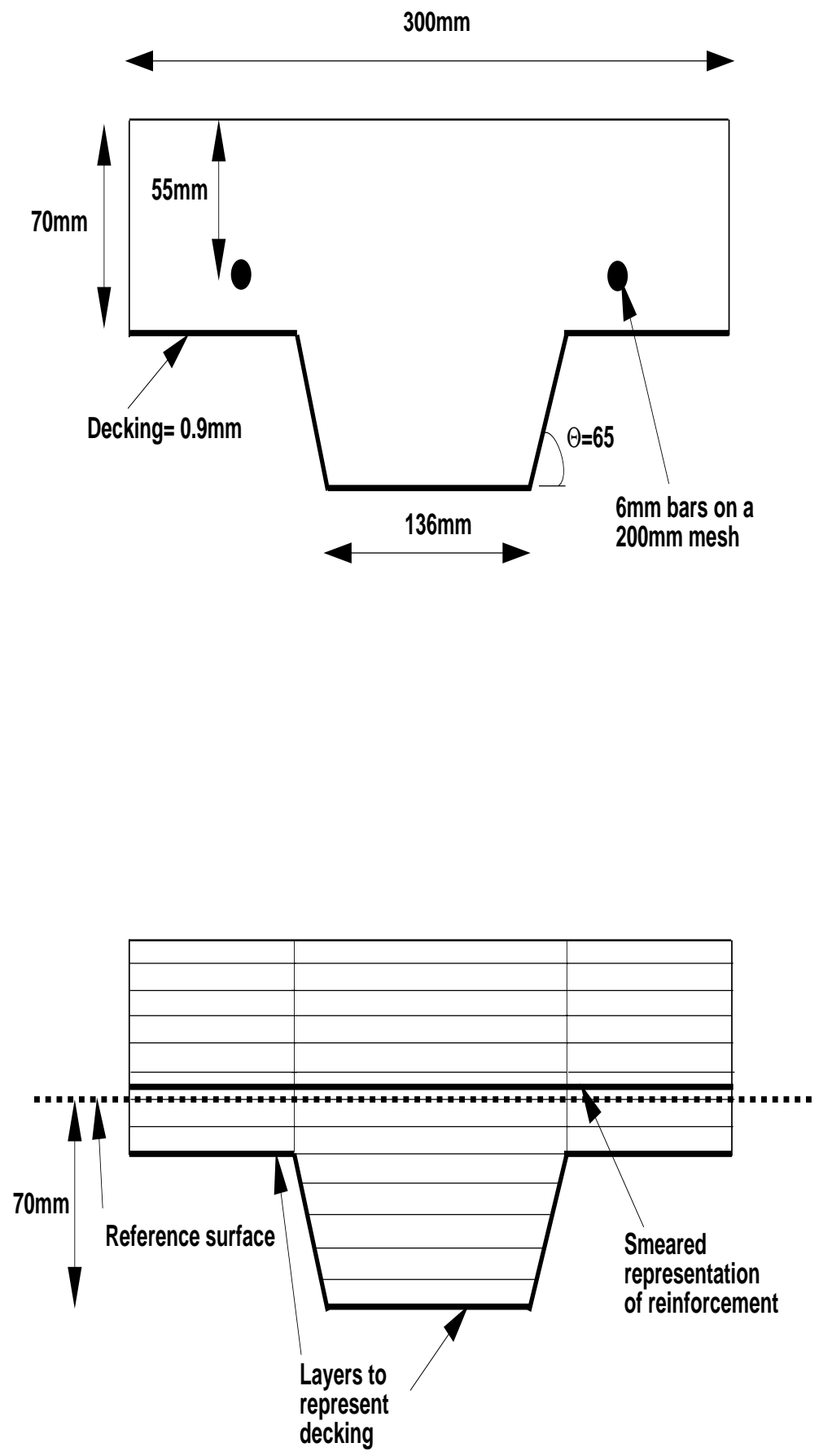

Figure 4: Diagrams showing the dimensions of one rib of the cross section of the Cardington slab and the division of the rib into layers 


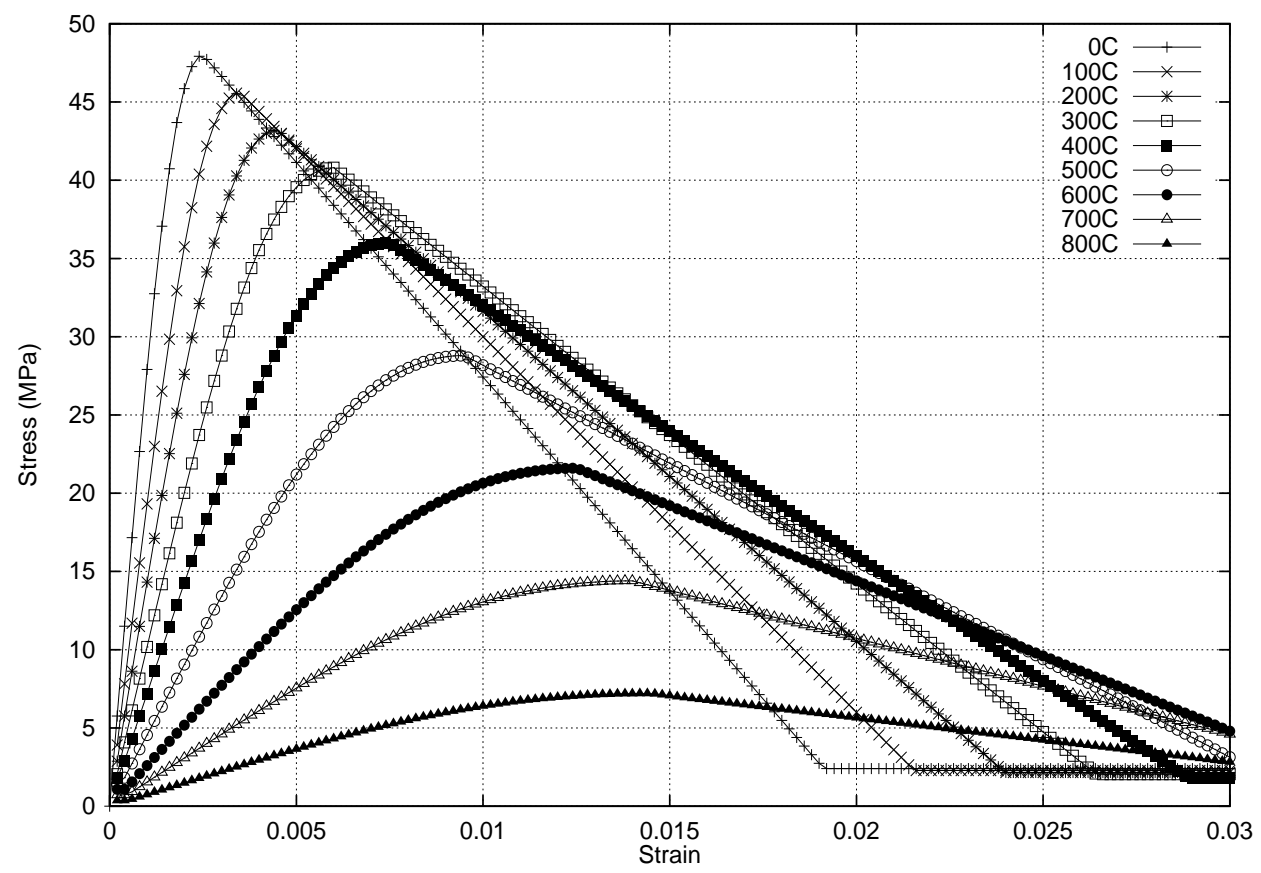

Figure 5: Compressive concrete material behaviour used by SRAS for modelling the Cardington slab 


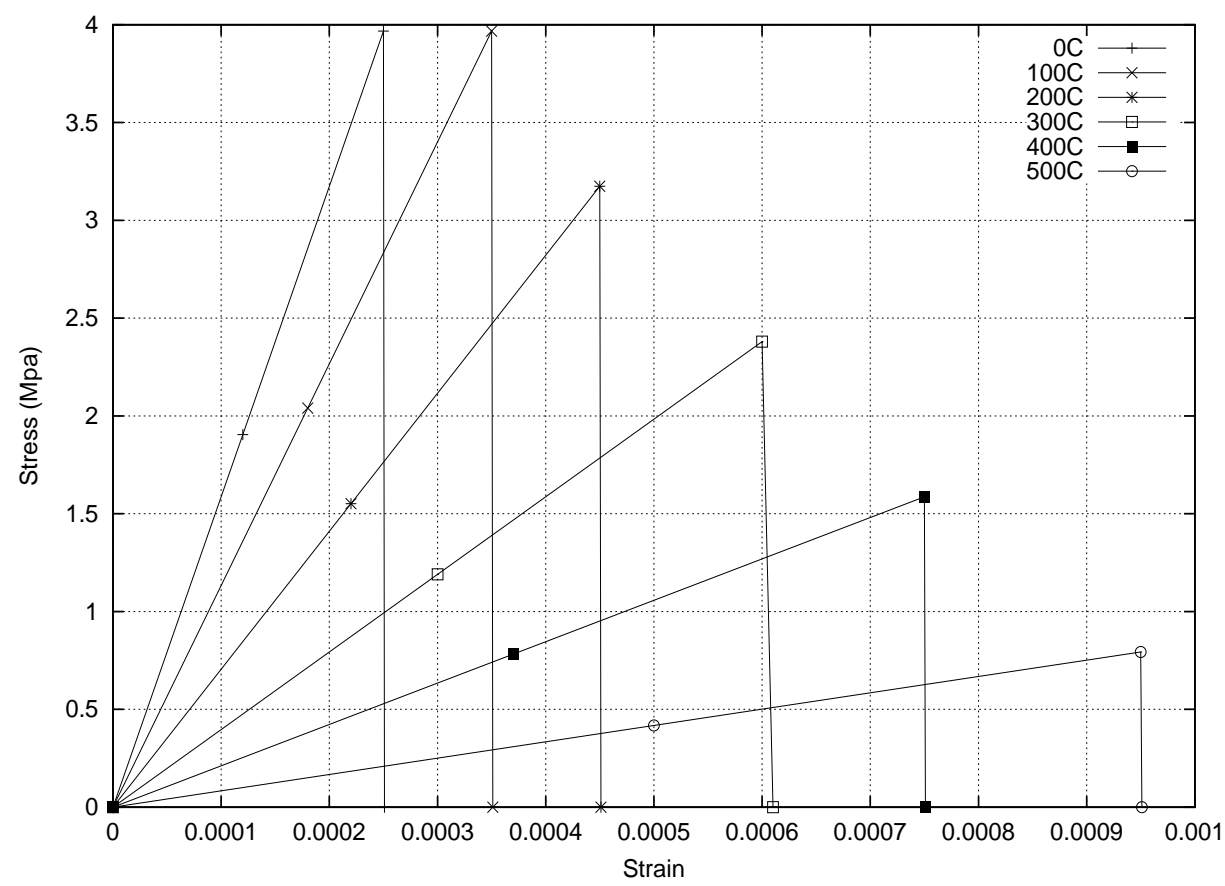

Figure 6: Tensile concrete material behaviour used by SRAS for modelling the Cardington slab 


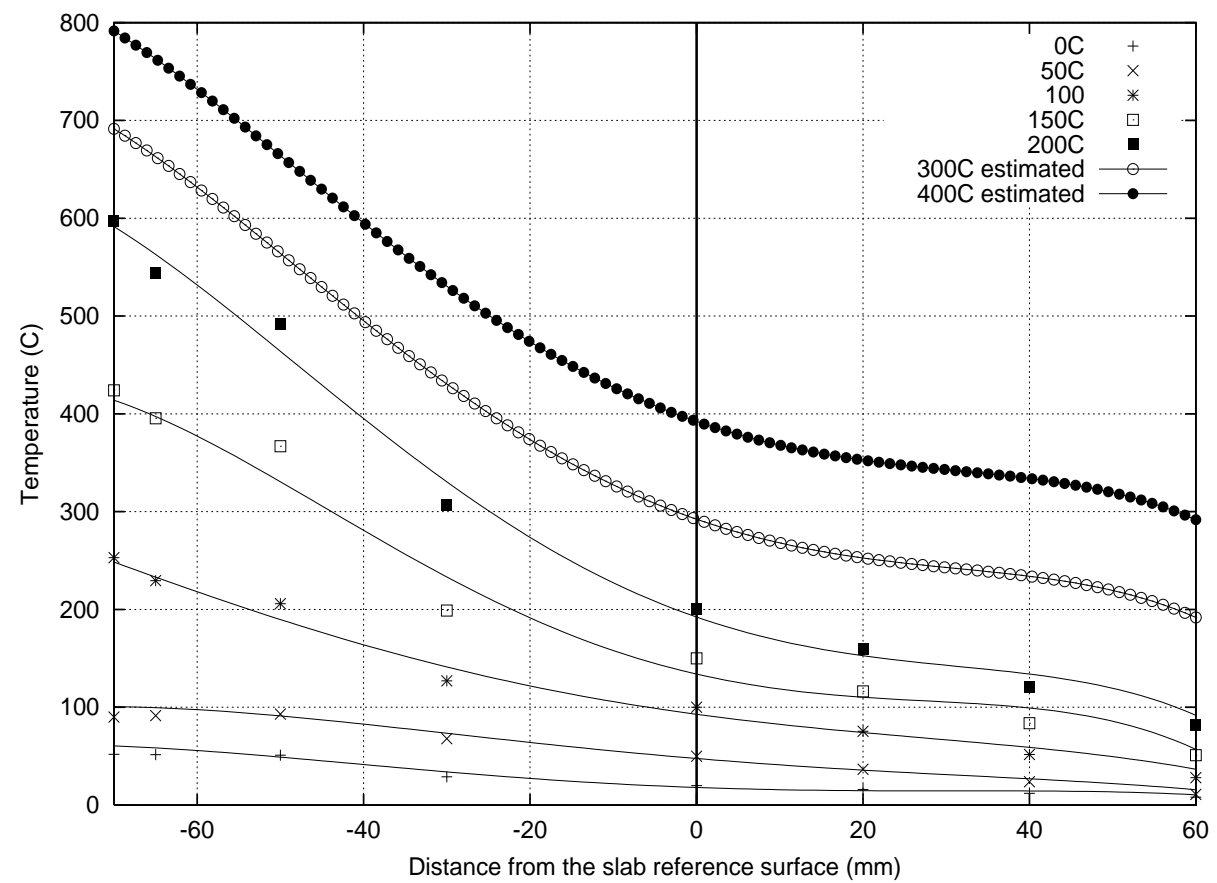

Figure 7: Temperature profiles through a rib section of the Cardington slab with polynomial curves fitted 


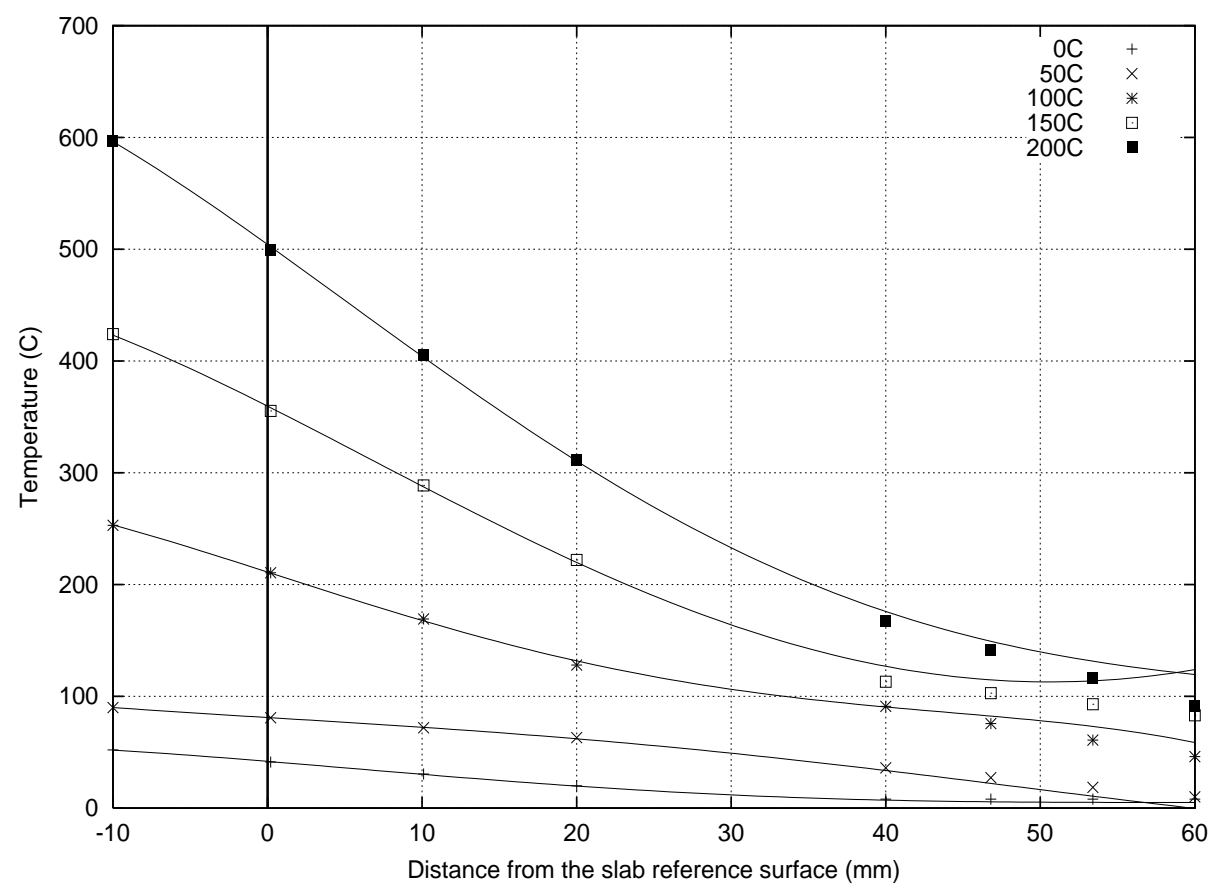

Figure 8: Temperature profiles through a trough section of the Cardington slab with polynomial curves fitted 


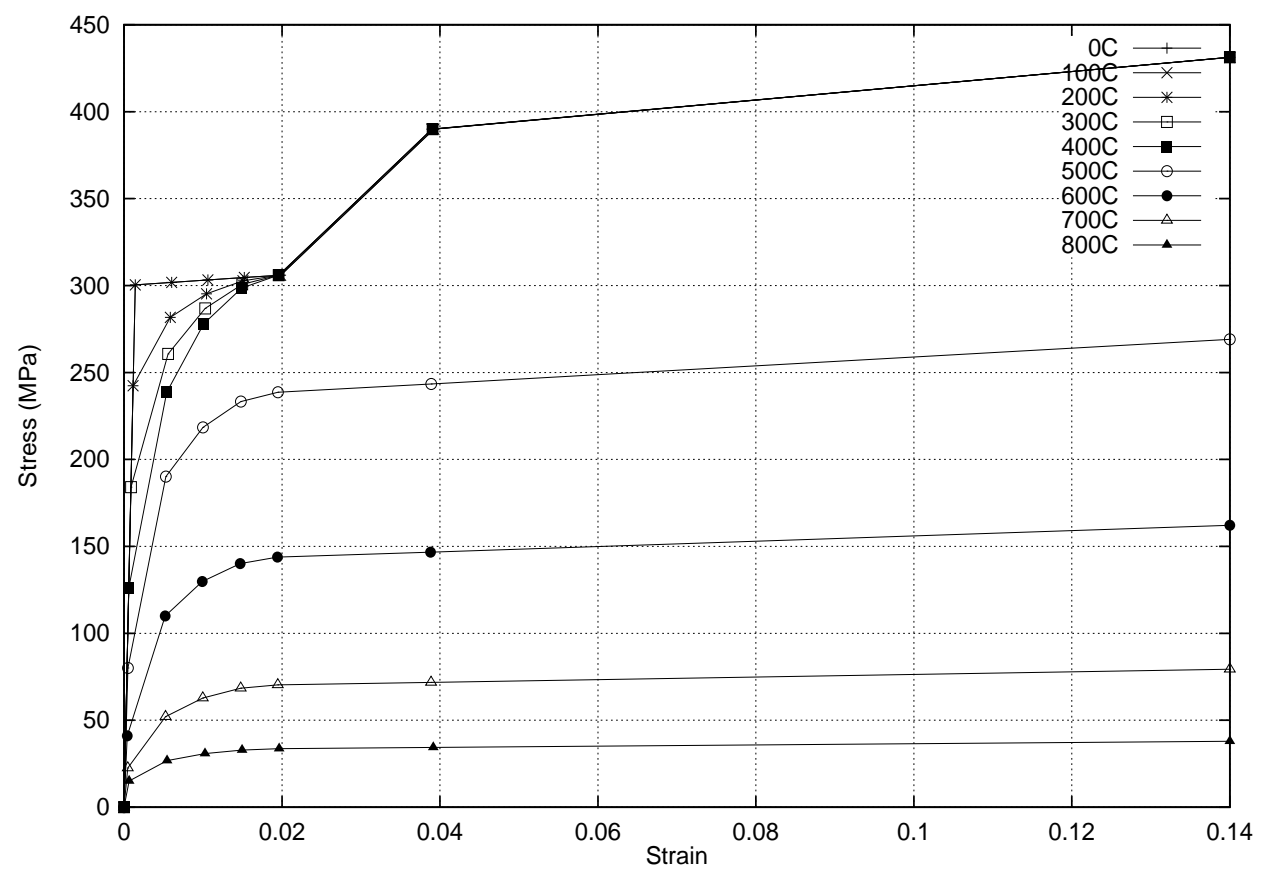

Figure 9: Steel material behaviour used by SRAS for modelling the Cardington slab 


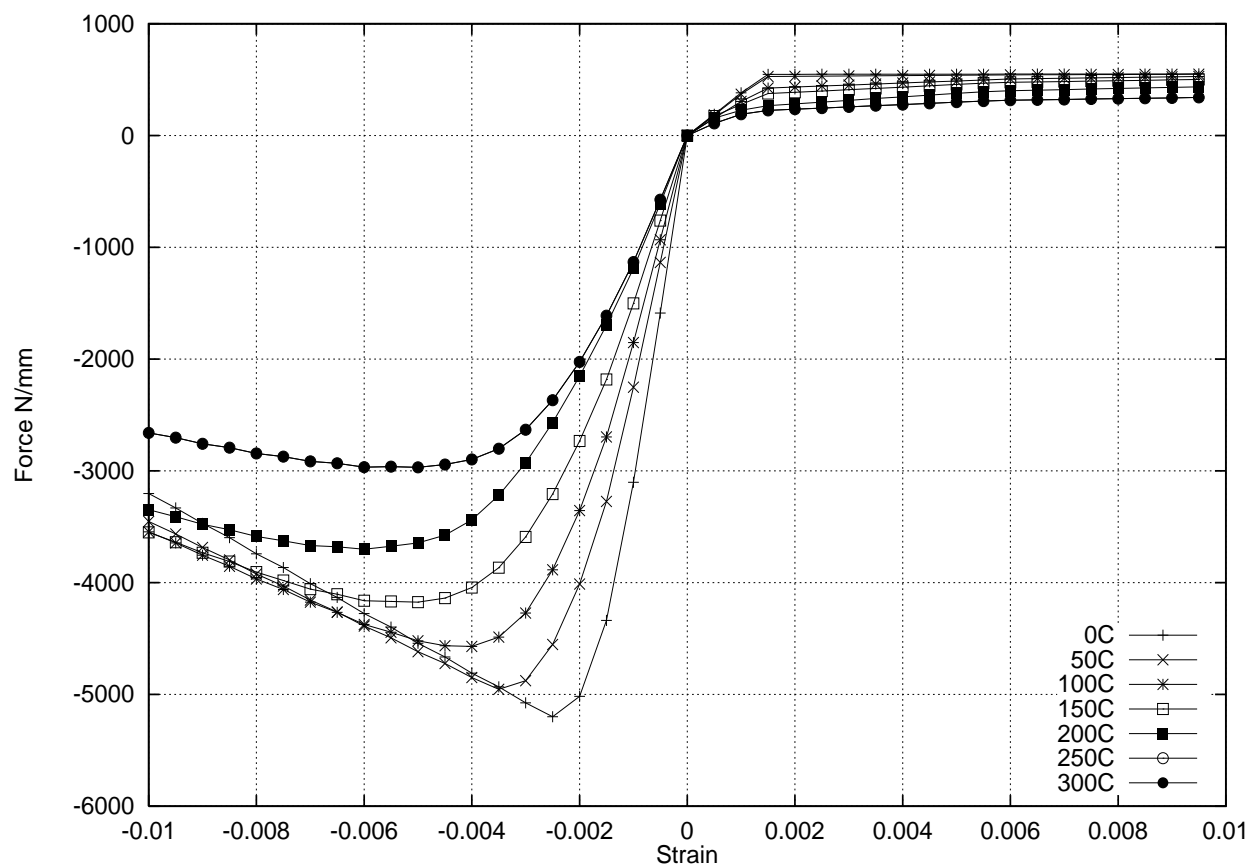

Figure 10: Force-strain diagram for the Cardington slab parallel to the ribs; zero curvature. 


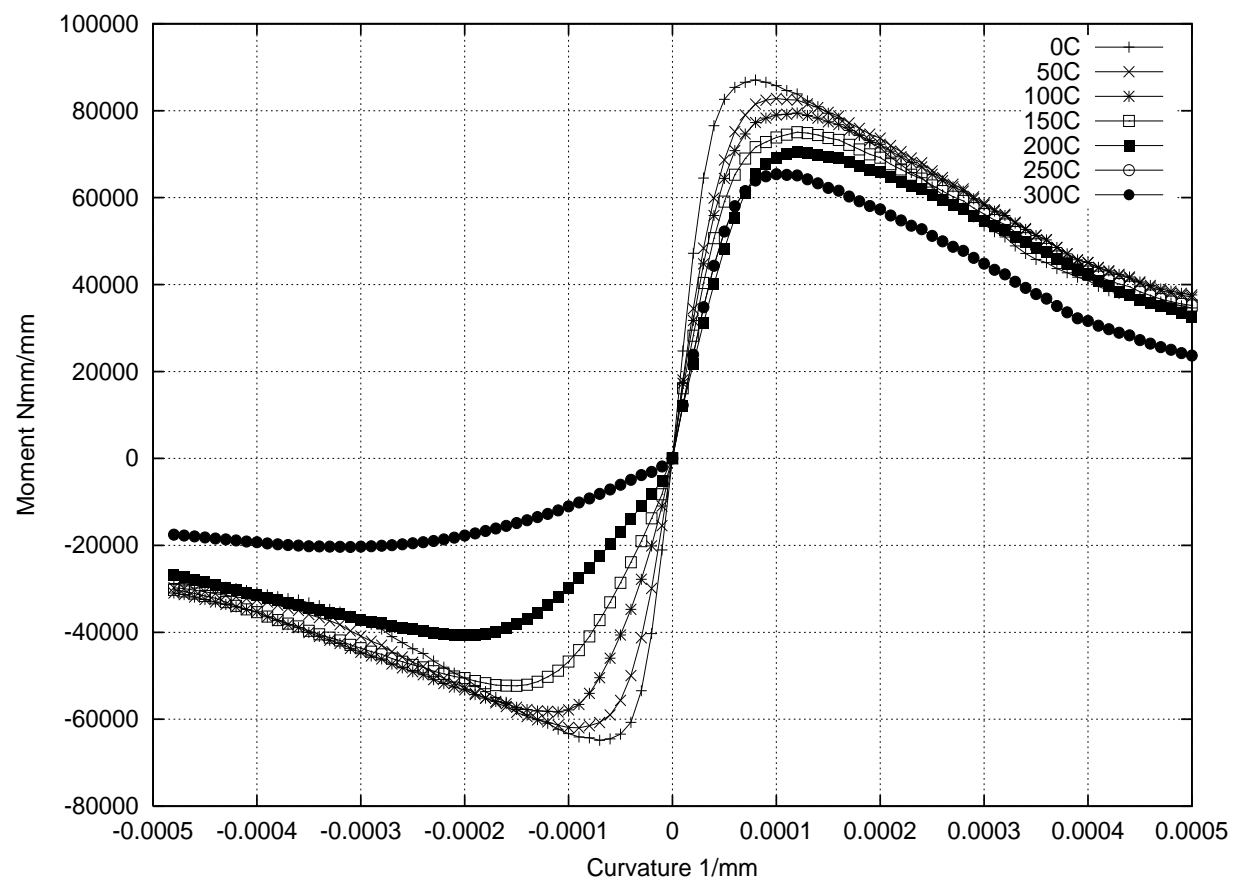

Figure 11: Moment-curvature diagram for the Cardington slab parallel to the ribs;zero strain. 


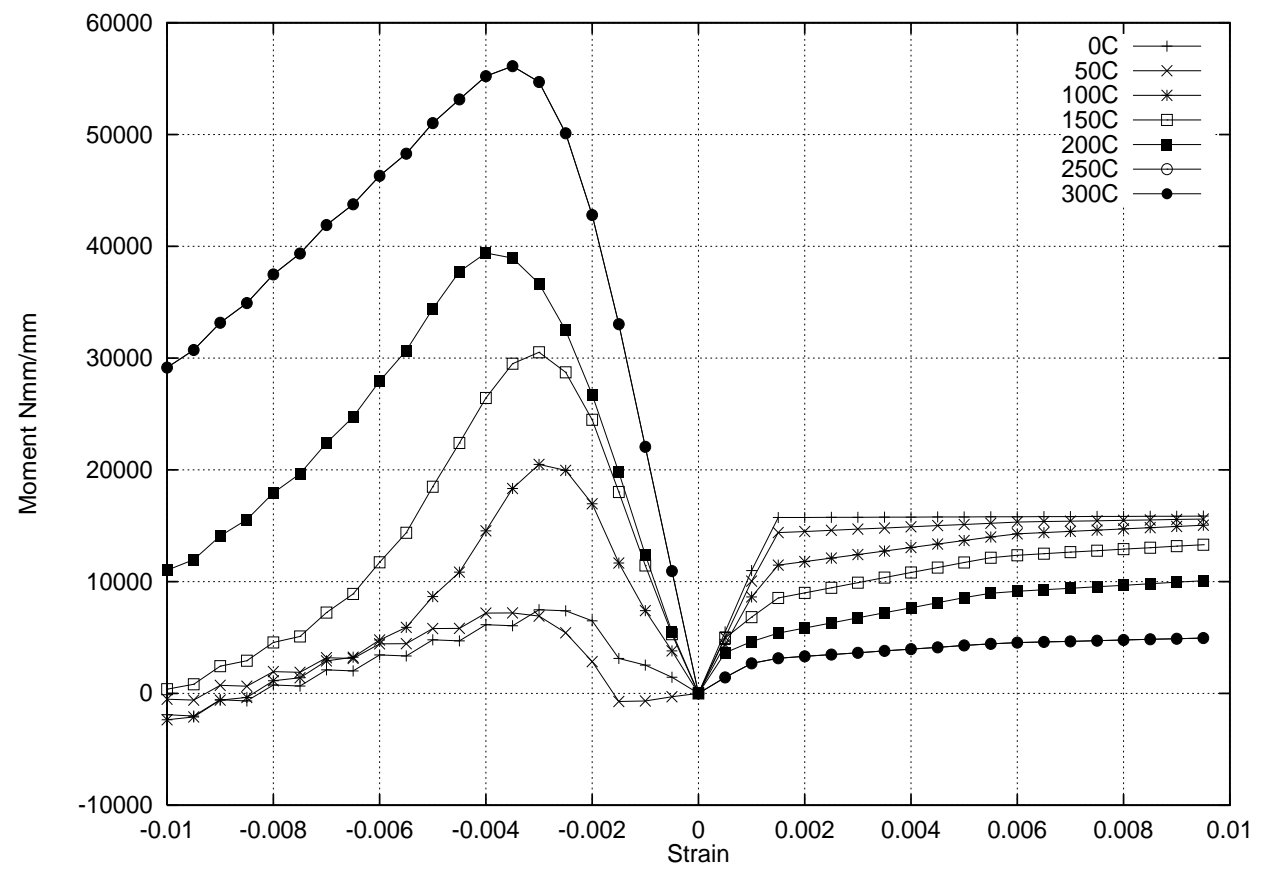

Figure 12: Moment-strain diagram for the Cardington slab parallel to the ribs; zero curvature. 


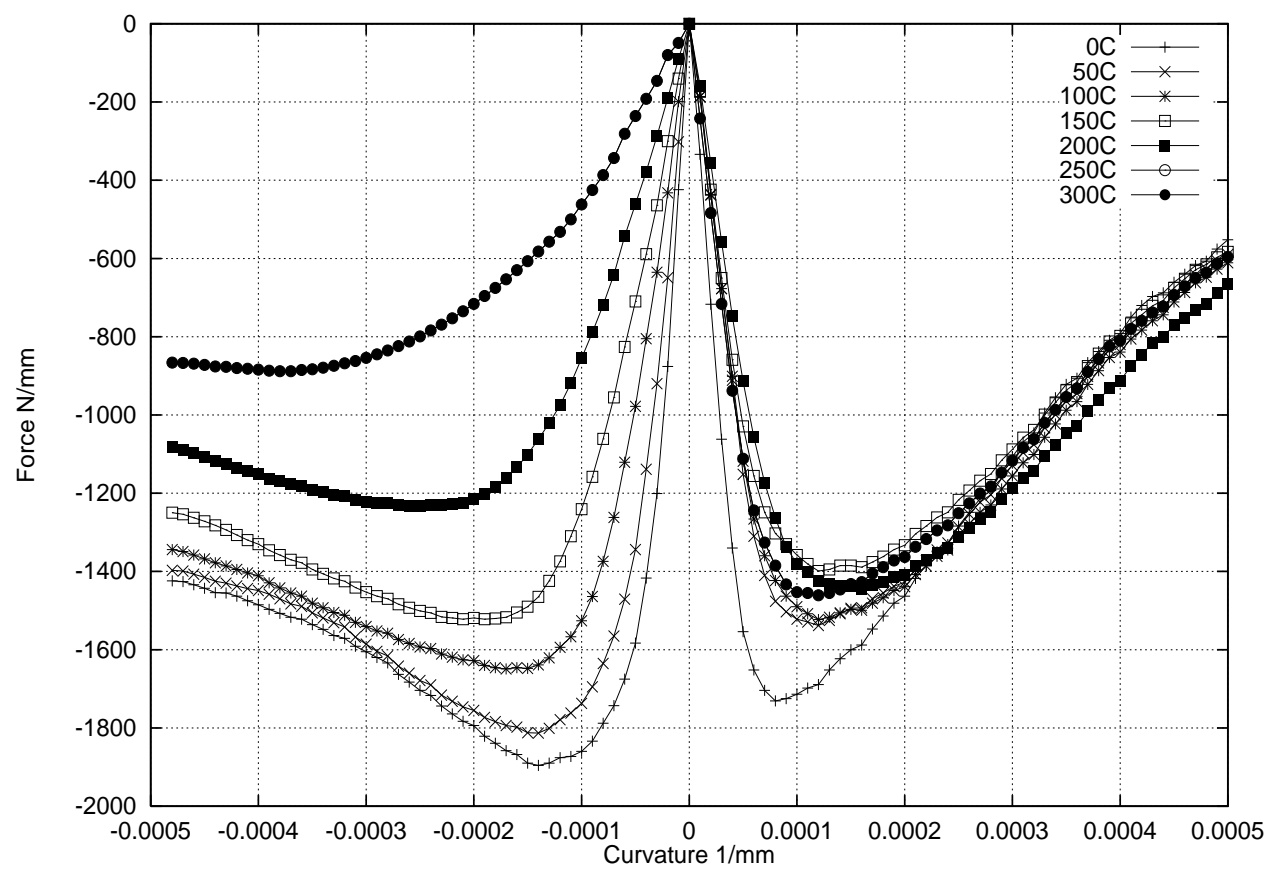

Figure 13: Force-curvature diagram for the Cardington slab parallel to the ribs; zero strain. 


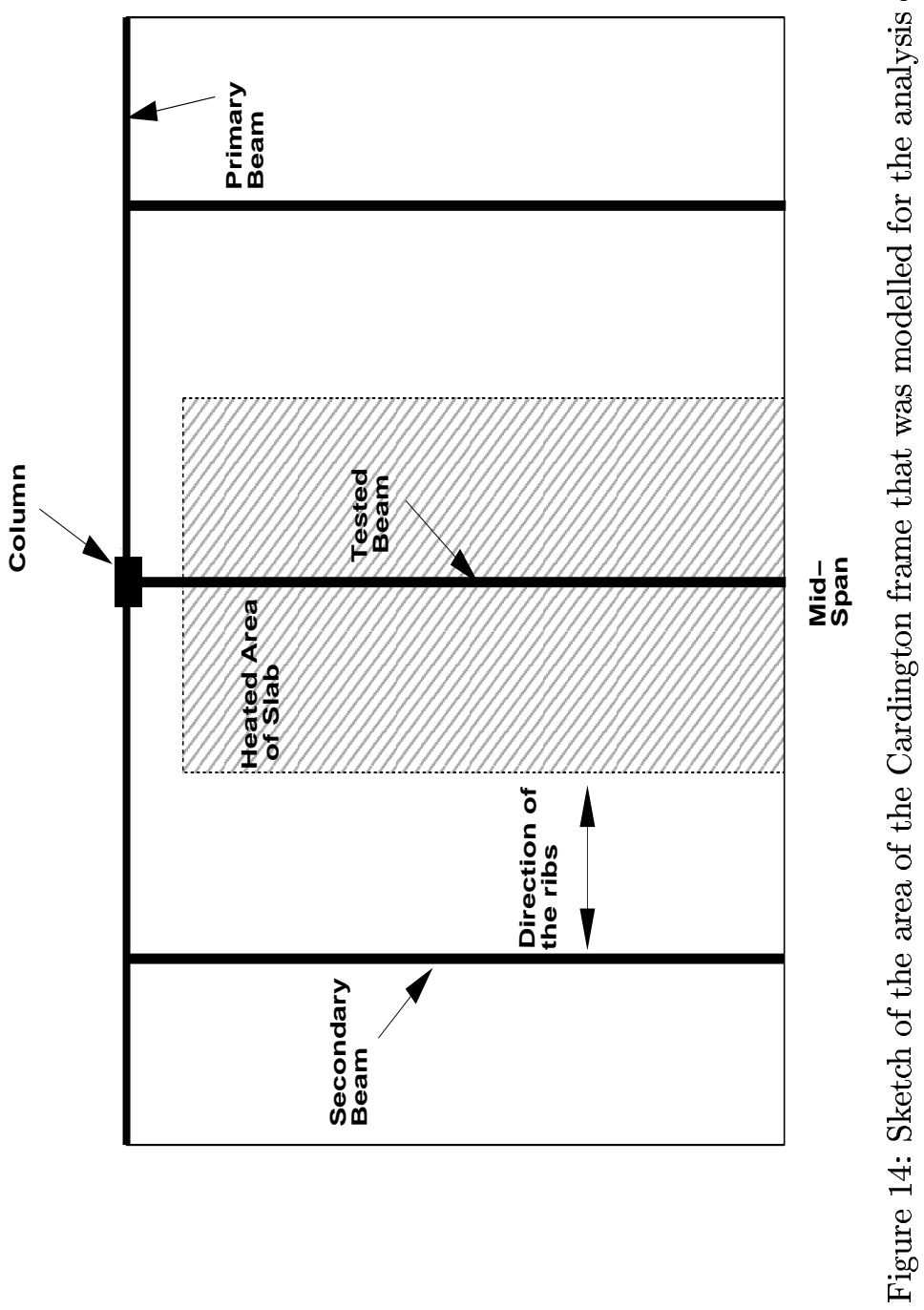




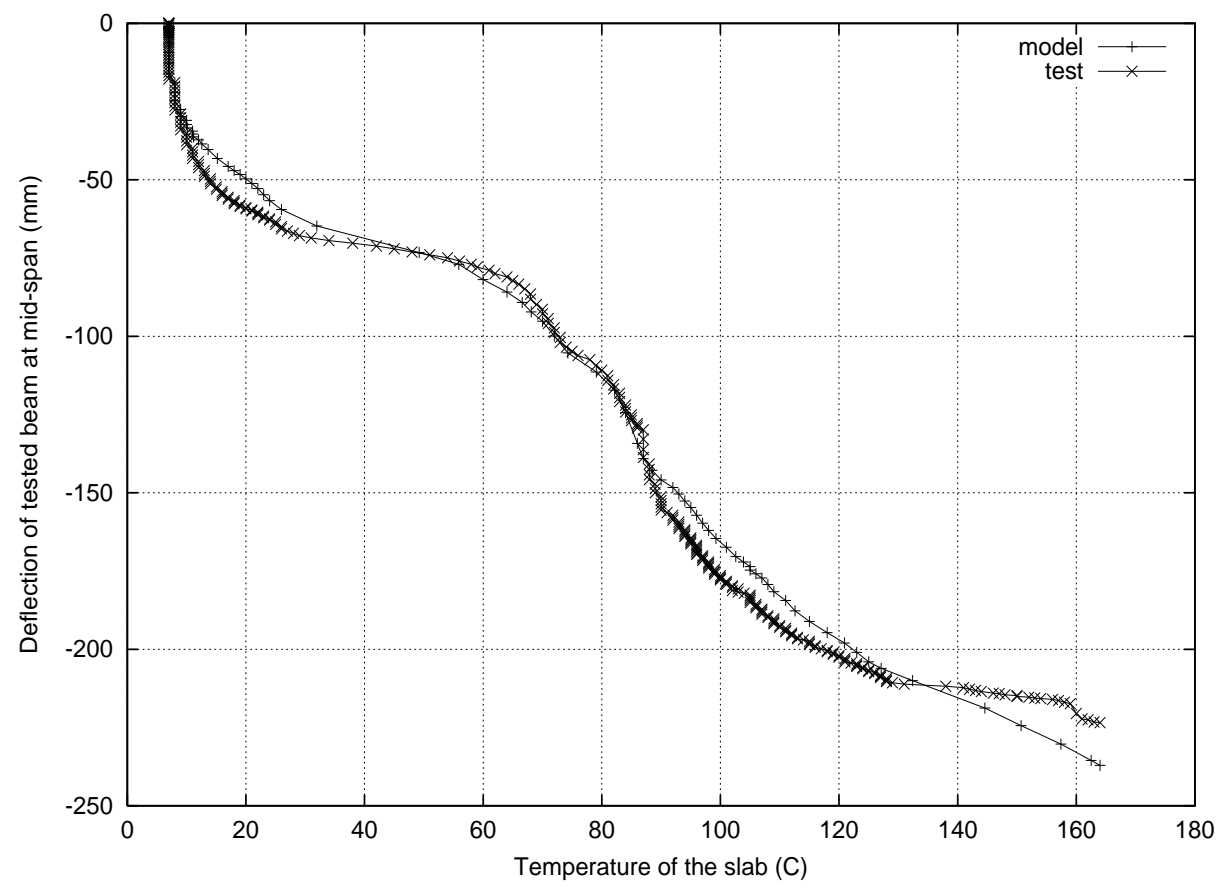

Figure 15: Comparison of the mid-span deflections from the experiment and numerical simulation for the first Cardington test 